\title{
Performance testing of collision-avoidance system for power wheelchairs
}

\author{
Edmund F. LoPresti, PhD; ${ }^{\text {* }}$ Vinod Sharma, PhD; ${ }^{2}$ Richard C. Simpson, PhD; ${ }^{2-3}$ L. Casimir Mostowy $^{1}$ \\ ${ }^{1}$ AT Sciences, LLC, Pittsburgh, PA; ${ }^{2}$ Department of Rehabilitation Science and Technology, University of Pittsburgh, \\ Pittsburgh, PA; ${ }^{3}$ Pittsburgh Highland Drive Department of Veterans Affairs Medical Center, Pittsburgh, PA
}

\begin{abstract}
The Drive-Safe System (DSS) is a collisionavoidance system for power wheelchairs designed to support people with mobility impairments who also have visual, upperlimb, or cognitive impairments. The DSS uses a distributed approach to provide an add-on, shared-control, navigationassistance solution. In this project, the DSS was tested for engineering goals such as sensor coverage, maximum safe speed, maximum detection distance, and power consumption while the wheelchair was stationary or driven by an investigator. Results indicate that the DSS provided uniform, reliable sensor coverage around the wheelchair; detected obstacles as small as $3.2 \mathrm{~mm}$ at distances of at least $1.6 \mathrm{~m}$; and attained a maximum safe speed of $4.2 \mathrm{~km} / \mathrm{h}$. The DSS can drive reliably as close as $15.2 \mathrm{~cm}$ from a wall, traverse doorways as narrow as $81.3 \mathrm{~cm}$ without interrupting forward movement, and reduce wheelchair battery life by only $3 \%$. These results have implications for a practical system to support safe, independent mobility for veterans who acquire multiple disabilities during Active Duty or later in life. These tests indicate that a system utilizing relatively low cost ultrasound, infrared, and force sensors can effectively detect obstacles in the vicinity of a wheelchair.
\end{abstract}

Key words: assistive technology, collision avoidance, intelligent mobility aids, man-machine systems, mobility impairments, navigation assistance, rehabilitation, robotics, smart wheelchairs, wheeled mobility.

\section{INTRODUCTION}

"Independent mobility is critical to individuals of any age. Children without safe and independent self-ambulation are denied critical learning opportunities, which places them at a developmental disadvantage relative to their self- ambulating peers”, [1-3]. “Adults who lack an independent means of locomotion are less self-sufficient, which can manifest itself in a negative self-image" ${ }^{\text {a }}$ [4]. "A lack of independent mobility at any age places additional obstacles in the pursuit of vocational and educational goals”a $[2,5]$. "While the needs of many individuals with disabilities can be satisfied with powered wheelchairs," “a significant number of individuals with disabilities are denied powered mobility because they lack the visual, motor, or cognitive skills required to operate a powered wheelchair safely"b [6]. "This population includes, but is not limited to, individuals with low vision, visual field neglect, spasticity, or tremors"a [7]. For example, "the American Federation for the Blind has estimated that 9.61 percent of all individuals who are legally blind also use a wheelchair or scooter,"b and an additional " 5.25 percent of individuals who have serious difficulties seeing (but are not legally blind), ${ }^{\text {b }}$ also use a wheelchair or scooter [7]. Although some people are able to use a cane or dog guide while using a wheelchair [8-9], many wheelchair users with visual impairments currently rely on an attendant for mobility [10].

To accommodate people who are not served by existing wheelchair technology, several researchers have used

\footnotetext{
Abbreviations: DSS = Drive-Safe System, IR = infrared, LED = light-emitting diode, ODM = obstacle density map, $\mathrm{SD}=$ standard deviation.

*Address all correspondence to Edmund F. LoPresti, PhD; AT Sciences, LLC, 4724 Coleridge St, Pittsburgh, PA 15201; 412-901-1042; fax: 412-781-3048.

Email: edlopresti@at-sciences.com

DOI:10.1682/JRRD.2010.01.0008
} 
mobile robotics technologies to create "smart wheelchairs" [11]. "A smart wheelchair typically consists of either a standard power wheelchair to which a computer and a collection of sensors have been added or a mobile robot base to which a seat has been attached."a

Many smart wheelchairs have used a combination of bumpers, infrared (IR) sensors, and sonar sensors for collision avoidance [12-14]. While these systems have often provided good results under controlled conditions, they have tended to lack sufficient sensor coverage to be useful in realistic settings. Further, smart wheelchairs (like many other mobile robots) frequently rely on odometry information from the wheels. For smart wheelchairs, this requires modifications to the drive wheels, which would be impractical in an aftermarket add-on device for consumers and clinicians.

Some smart wheelchairs achieve safer navigation by using a laser range finder along with sonar and IR sensors for more reliable and comprehensive sensor coverage [15-16]. Laser range finders can further assist in drop-off detection. However, the cost of the laser range finder and associated hardware is prohibitively high for a commercially viable smart wheelchair. Other smart wheelchairs use stereo vision cameras for obstacle and drop-off detection and localization [17]. While promising, this technology is relatively new and has not been tested fully.

Some smart wheelchairs provide point-to-point navigation by following a colored lane, a magnetic ferrite marker track, or a barcode [18]. While useful in some settings, lanefollowing technology requires significant modifications to the user's environment and once the wheelchair leaves the marked path, navigation assistance is no longer available.

The system described in this article builds on prior work toward the development of a smart wheelchair system that provides collision avoidance at a low cost with minimal modifications to the underlying wheelchair or environment [12,19-20]. "The Drive-Safe System (DSS) is an add-on, distributed, shared control navigation assistance system for powered wheelchairs, intended to provide safe and independent mobility”b (Figure 1). The "user is responsible for high-level control of the system, such as choosing the destination, path planning, and some navigation actions, while the DSS overrides unsafe maneuvers through autonomous collision avoidance, automatic wall following, and door crossing." In this project, we redesigned the DSS from earlier prototypes to achieve a more robust, commercially viable design.

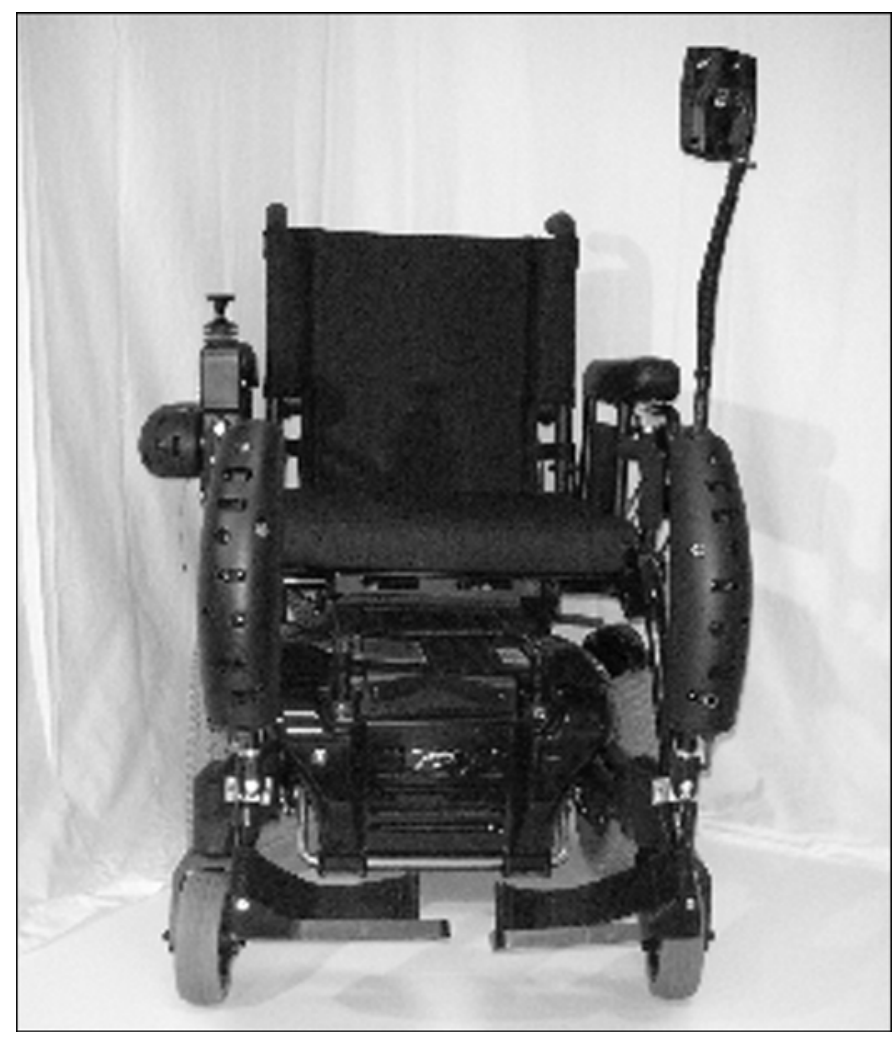

Figure 1.

Wheelchair equipped with Drive-Safe System.

The goal of this study was to answer several questions related to the safety and practicality of the current prototype:

- Can the DSS detect obstacles it is approaching in any direction?

- How close must an obstacle be for the DSS to detect it?

- When the wheelchair is driving down a corridor, how narrow can the corridor be before the DSS detects both walls as obstacles and stops the wheelchair?

- When the wheelchair is traversing a doorway, how narrow can the doorway be before the DSS detects both edges of the door as obstacles and stops the wheelchair?

- How quickly can the wheelchair drive and still stop in advance of an obstacle?

- How much pressure is required on the bumper to trigger the bumper sensor and stop the wheelchair?

- To what extent will the extra power drain of the DSS limit the range of the wheelchair?

Note that in this testing, the focus was on determining the performance parameters of the prototype rather than statistically proving a hypothesis. Testing of the efficacy of the system as a tool for people with mobility impairment will be hypothesis driven. 


\section{METHODS}

\section{System}

\section{Hardware}

The "DSS has a modular architecture, with a central control and interface node (the joystick translator) communicating with one or more sensor nodes"b (Figure 2). "The sensor nodes monitor the environment for potential obstacles and deliver auditory and visual alerts to the user."b The modular architecture allows the system to use more or fewer sensor nodes depending on the user's needs, for example, only providing coverage on one side of the wheelchair if a user has a loss of vision on that side. In the testing described subsequently, the DSS had five sensor modules providing coverage on all sides of the wheelchair.
The "joystick translator communicates with the wheelchair to determine the desired direction of travel and to override the"b user's control when a potential collision is detected. The joystick translator receives sensor information from the sensor nodes and commands the sensor nodes regarding which auditory or visual alerts to present to the user. The joystick translator and sensor nodes communicate using a Controller Area Network (CAN 2.0).

The DSS "is designed to act as a specialty user interface, allowing it to connect with the control electronics of a variety of wheelchairs. The current DSS prototype has been tested on wheelchairs from Pride Mobility (http:// www.pridemobility.com/) and Sunrise Medical (http:// www.sunrisemedical.com/), and past prototypes were tested on wheelchairs by Invacare (http://www.invacare.com/), Permobil (http://www.permobil.com/), and Everest and

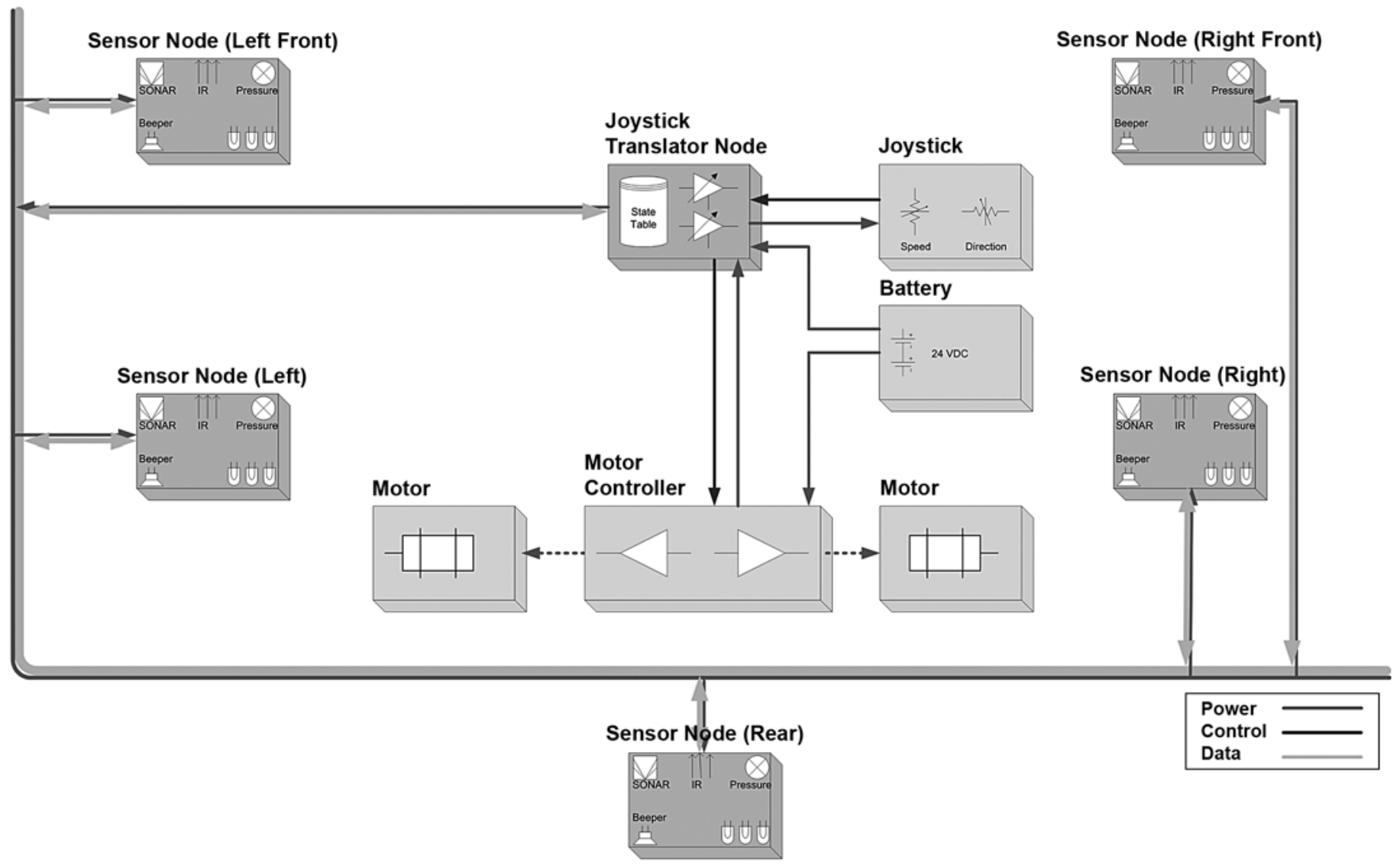

Figure 2.

Drive-Safe System (DSS) block diagram. DSS sensor nodes communicate with DSS joystick translator node, which in turn "communicates with the wheelchair joystick (to obtain desired direction of travel)" (Simpson RC, LoPresti EF, Cooper RA. How many people would benefit from a smart wheelchair? J Rehabil Res Dev. 2008;45(1):53-71. [PMID: 18566926] DOI:10.1682/JRRD.2007.01.0015), wheelchair batteries (to obtain power), and wheelchair motor controller (to control direction of travel). IR = infrared, LED = light-emitting diode. 
Jennings (http://www.grahamfield.com/). The wheelchair joystick (or other control device) plugs into the joystick translator, and the joystick translator plugs into the wheelchair's motor controller (Figure 2). The joystick translator also obtains power from the wheelchair batteries, and provides power to the sensor nodes.

"Each sensor node consists of five ultrasonic rangefinders (sonars), five IR rangefinders, two bumper inputs, one speaker, and three status light-emitting diodes (LEDs).", Three different sonars are used: LV-MaxSonar-EZ1, LVMaxSonar-EZ2, and LV-MaxSonar-EZ3 (MaxBotix Inc; Brainerd, Minnesota, http://www.maxbotix.com/). All sonars report the distance to the nearest obstacle for obstacles between 15 and $645 \mathrm{~cm}$ from the sensor. The three sonar models vary in the width of their detection cone and were selected for different positions around the wheelchair to maximize sensor coverage and minimize cross talk between sensors. The sonar with the widest cone, EZ-1, was used in some slots of the two front-corner nodes and the rear node to provide wide coverage in areas not covered by other nodes. The EZ-2 was used in some slots of the front-corner and side nodes to reduce cross talk in areas covered by multiple nodes. The sonar with the narrowest cone, EZ-3, was used in some slots for all five nodes to provide more precise coverage in areas that might be used to distinguish objects such as door frames.

Two types of IRs are used in the current DSS architecture: IR-GP2Y0A02YK and IR-GP2D120 (Sharp Corporation; Osaka, Japan, http://sharp-world.com/). The GP2Y0A02YK provides range information from 20 to $152 \mathrm{~cm}$ with $2.5 \mathrm{~cm}$ resolution, while the GP2D120 provides range information from 3.8 to $30.5 \mathrm{~cm}$ with $2.5 \mathrm{~cm}$ resolution. The GP2Y0A02YK was used in all nodes to provide secondary coverage of relatively distant obstacles. The GP2D120 was used in the side nodes to provide coverage of nearby obstacles, such as when the wheelchair is closely following a wall.

Each sensor node has two force sensing resistors (Interlink Electronics; Camarillo, California, http:// www.interlinkelectronics.com/) that exhibit a decrease in resistance when increased pressure is applied to the surface of the sensor. When an obstacle touches a bumper segment, the sensor node transmits the position of the obstacle to the translator node.

Each sensor node has three status LEDs and a speaker that provide visual and auditory feedback to the user. The pattern of LEDs and the pattern of tones emitted through the speakers indicate the status of the system and the presence of obstacles. The auditory feedback, together with the behavior of the wheelchair, is intended as the primary feedback for the user. The sensor nodes are equipped with LEDs primarily as a diagnostic tool for maintenance and are further used as an additional form of user feedback. The LEDs are placed so that a wheelchair driver with normal visual acuity can see the patterns of the LEDs on the front and side sensor nodes.

\section{Software}

The DSS software is distributed across the sensor nodes and the joystick translator node. The "translator node plays the central role in implementing collision avoidance.”b The translator node's function is to intercept the user's joystick signals and send a modified joystick signal to the wheelchair motor controller. The translator "maintains the current state of each sensor node element (e.g., sonar range information, IR range information, bumper state, state of the ${ }^{\text {,b }}$ speaker and LEDs) based on data from the sensor nodes. Sensor data are used to check for the presence of obstacles in the direction the user is pointing the joystick. The state of the speakers and LEDs are tracked so that the translator can update them as necessary to issue (or cancel) warnings.

"Typically, the translator provides a signal to the motor controller that is identical to the original joystick signal, preserving the user's control. However, if the system detects a collision risk, the translator will scale the signal (slowing the wheelchair) or send a neutral signal (stopping the wheelchair). The translator does not vary the direction of travel from the user's original intent." "b The specific behavior of the system depends on the operating mode. The DSS implements five operating modes: obstacle-avoidance mode, door-crossing mode, wall-following mode, corridorcrossing mode, and override mode.

In obstacle-avoidance mode, the area around the wheelchair is divided into 16 sectors (Figure 3). Each sector relates to an intended direction of movement of the wheelchair based on the joystick position. For example, pushing the joystick between $67.5^{\circ}$ and $90^{\circ}$ will activate sector 2. Each sector is associated with one or more sonar and IR sensors (and a given sensor may cover multiple sectors). For a given sector, each associated sensor reading is assigned to one of three regions (Figure 3):

- Safe Region: The safe region is the region farthest away from the wheelchair. Having an obstacle in this region will not affect the movement of the wheelchair in this sector. 


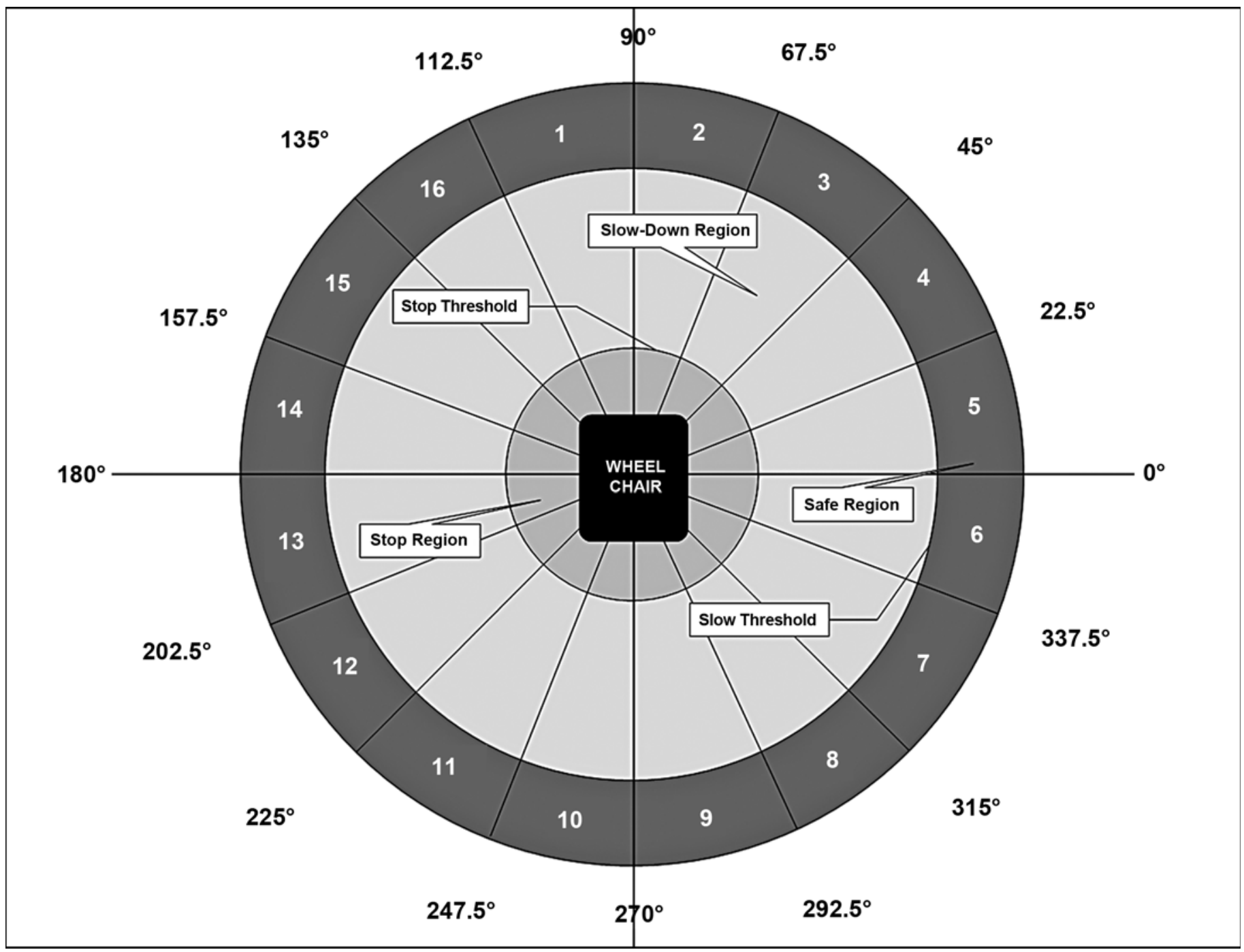

Figure 3.

Wheelchair sector information. Front of wheelchair is at $90^{\circ}$, left side at $180^{\circ}$.

- Slow Region: An obstacle in the slow region in a given sector will reduce the speed of the wheelchair in that sector, but the direction of the movement will remain the same. The rate of slowing down is proportional to the speed of the wheelchair and the minimum obstacle distance.

- Stop Region: The stop region is nearest to the wheelchair. An obstacle in this region in a given sector will stop the movement of the wheelchair in that sector. The stop threshold varies from sector to sector and is larger in the front sectors $(1-4,15-16)$ than in the rear sectors (8-11) because the forward speed is typically higher than the reverse speed and a larger stop threshold pro- vides enough distance for the wheelchair to stop after signals are sent to the wheelchair controller.

The translator node gathers information from all the sensor nodes and organizes this information in an obstacle density map (ODM) database. The translator samples the joystick at $20 \mathrm{~Hz}$, and the joystick signal is analyzed to determine the sector where the driver intends to move. Based on the intended direction of movement and obstacle density from the ODM in that sector, the translator can make three choices:

1. Do not change the input speed and direction signals if there are no obstacles or obstacles are in the safe region. 
2. Slow down the wheelchair if obstacles are in the slow down region.

3. Stop the wheelchair if the obstacles are in the stop region.

The bumper modules provide additional protection in case the sonar and IR sensors fail to detect an obstacle. When a bumper segment is pressed, the DSS will not allow movement in the sectors covered by the pressed bumper.

Whenever the translator stops the wheelchair because of an obstacle, users are notified through auditory and visual feedback. The user has the option to steer away from the obstacle in the direction of any clear sector, allowing the user to maneuver around the obstacle and continue moving.

The override mode reduces wheelchair control signals to 35 percent of the actual joystick values without regard to data from the sonar or IR sensors and only stops the wheelchair in response to the bumper sensors. This mode allows the user to get close to something (e.g., desk, water fountain, light switch) without the DSS stopping the wheelchair. The user initiates override mode by pressing a switch. Users are notified when the DSS is in override mode by a distinct sound pattern from the sensor nodes. Users can revert to the normal obstacle-avoidance mode by pressing the override switch again.

The door-crossing, wall-following, and corridorcrossing modes assist with specific goals. The obstacleavoidance mode can make it difficult for users to pass through doorways or narrow corridors because the doorway or corridor wall may be confused with an obstacle. In obstacle-avoidance mode, each time the translator node creates the ODM, it checks whether the ODM matches any of the patterns defined in Table 1. If a pattern match is obtained, then the DSS enters the appropriate mode. For each of these modes, wheelchair speed is reduced to 75 percent and the bumpers are kept active. When the pattern match no longer applies, the DSS automatically returns to normal obstacle-avoidance mode.

\section{Performance Evaluation}

This study included seven subsections: sensor coverage, maximum obstacle-detection distance, minimum corridor width, minimum door width, maximum safe speed, bumper sensitivity, and power consumption. For clarity, the protocol for each subsection is included with the results for that subsection. All performance testing was performed by members of the research team, none of whom have motor impairments or uncorrected visual impairments.

\section{RESULTS}

\section{Sensor Coverage}

\section{Method}

Sensor coverage was tested with a Sunrise Rhythm Power Wheelchair and a Pride Mobility Q600 Power Wheelchair. Each wheelchair was placed in the middle of a $2.4 \times 2.4 \mathrm{~m}(8 \times 8 \mathrm{ft})$ grid. Each block in the grid was $5.1 \times$ $5.1 \mathrm{~cm} \mathrm{(2 \times 2} \mathrm{in.)} \mathrm{for} \mathrm{a} \mathrm{total} \mathrm{of} \mathrm{2,304} \mathrm{blocks,} \mathrm{of} \mathrm{which} \mathrm{the}$ central 432 blocks were occupied by the wheelchair.

To determine baseline sensor values, we recorded 20 samples of range data from each sensor without any obstacles in the grid. The mean and standard deviation (SD) of these 20 samples were calculated for each sensor. These mean values were used as baselines for the obstacle detection capability of the sensors.

Next, a $2.5 \mathrm{~cm}$ (1 in.) diameter by $1.5 \mathrm{~m}$ (5 ft) long wooden rod, fixed vertically on a stable flat surface, was used as an obstacle. The obstacle was consecutively placed in each of the 1,872 open blocks in the grid starting from the top-left corner of the grid. For each block, range data were recorded from all sensors. Throughout this testing, the wheelchair was stationary.

MATLAB 8.0 (MathWorks Inc; Natick, Massachusetts, http://www.mathworks.com/) was used for data analysis. For a given obstacle position in the grid, if the range value from at least one sensor was less than that sensor's baseline mean minus $3 \mathrm{SD}$, that grid position was considered "covered" by that sensor. Each grid cell could be covered by at least one sonar, at least one IR, at least one sonar and at least one IR, or no sonar or IR.

\section{Results}

Figure 4 shows the sensor coverage around the Pride Mobility wheelchair. Areas in the front of the wheelchair have coverage from both sonars and IRs, but there are blind spots in the front-right and rear corners of the wheelchair.

Originally, there was no coverage in the middle of the right and left sides of the wheelchair (as shown in Figure 4). Because the wheelchair is unable to move in those directions, sensor coverage was considered a low priority. However, initial testing with the DSS showed that having more coverage on the right and left sides of the wheelchair would support the door-crossing and wall-following modes. Therefore, the sensor coverage was modified to increase coverage on the right and left sides of the wheelchair. The results of 
Table 1.

Drive-Safe System (DDS) modes of operation. Mode of operation is determined by pattern of certain sectors being in stop region while certain other sectors are in slow and safe regions. Relevant sectors are illustrated by white segments and indicated by sector numbers as described in Figure 3. For example, DSS will enter front-door crossing mode (row 1) if sectors 4 and 15 are in stop region, sectors 3 and 16 are in slow region, but sectors 1 and 2 (directly in front of wheelchair) are in safe region, and joystick indicates travel toward sector 1 or 2 .

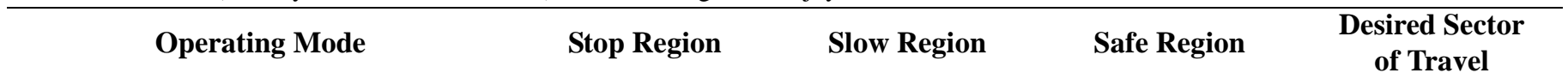

Front Door-Crossing

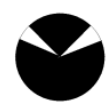

4,15

Rear Door-Crossing
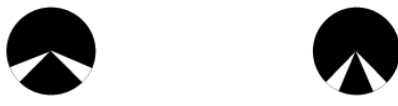

7,12

8, 11

9,10

9,10

Left Wall-Following Moving Forward
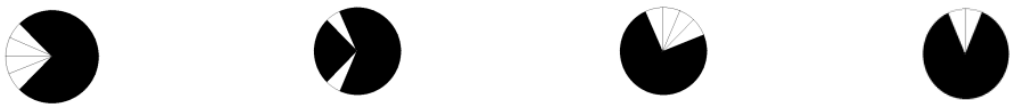

$12,13,14,15$

11,16

$1,2,3,4$

1,2

Left Wall-Following Moving Reverse
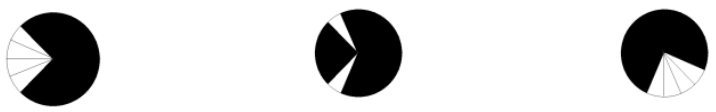

$12,13,14,15$

11,16

$7,8,9,10$

9,10

Right Wall-Following Moving Forward

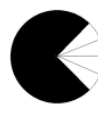

$4,5,6,7$

3, 8

$1,2,15,16$

1,2

Right Wall-Following Moving Backward

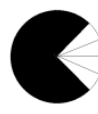

4, 5, 6, 7

3, 8

$9,10,11,12$

9,10

Corridor-Crossing Moving Forward
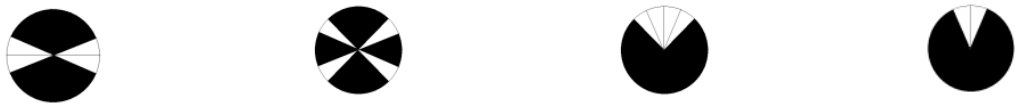

$5,6,13,14$

4, 7, 12,15

$1,2,3,16$

1,2

Corridor-Crossing Moving Backward
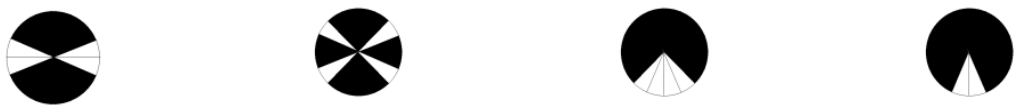

$5,6,13,14$

$8,9,10,11$

9,10 


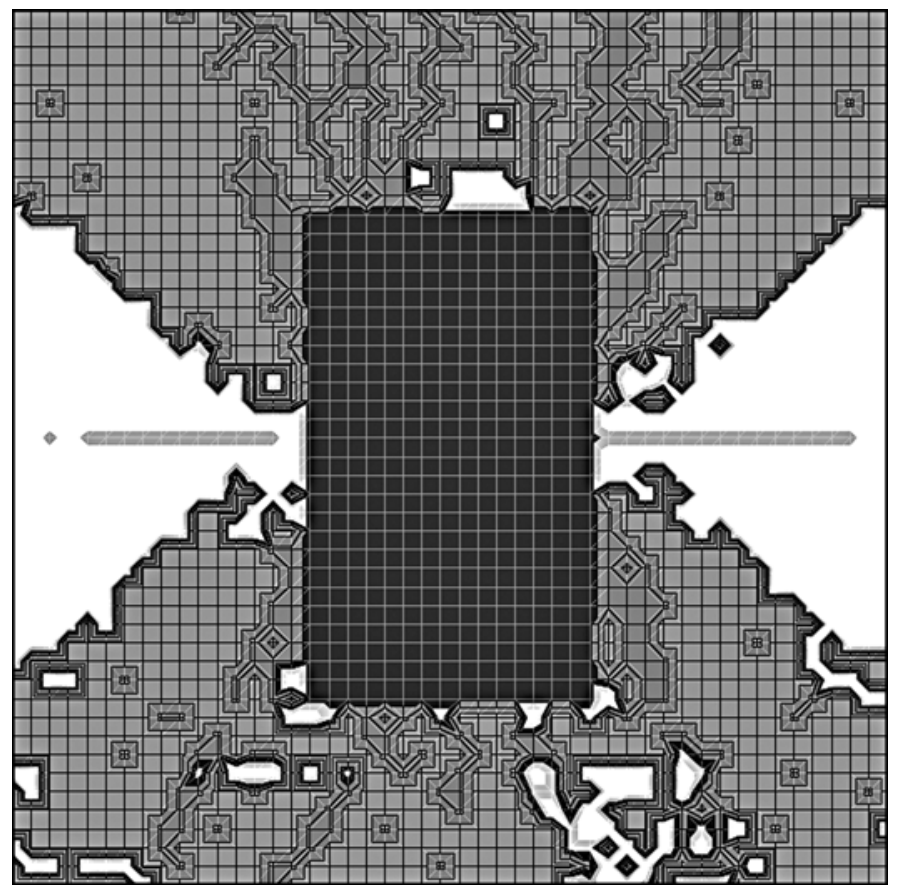

Figure 4.

Sensor coverage (Pride Mobility Wheelchair). Areas in dark gray are covered by at least one sonar and at least one infrared sensor (IR), medium gray by at least one sonar (but no IRs), and light gray by at least one IR (but no sonars). Areas in white are not covered by any sonar or IR. Black rectangle at center represents wheelchair.

testing with this modified sensor coverage for the Pride Mobility wheelchair are shown in Figure 5. Similar results were obtained for the Sunrise wheelchair.

\section{Maximum Obstacle-Detection Distance}

\section{Method}

The MaxSonar EZ1 (which has the widest detection cone of the sonars on the DSS) and the Sharp GP2Y0A02YK (which has the greatest range of the IRs on the DSS) were used in these tests. Cardboard and wooden cylinders of varying diameter were placed $7.6 \mathrm{~m}(25 \mathrm{ft})$ away from the front-right sensor node of the DSS and then brought closer to the sensor node until they were detected by the individual sensor being studied (ignoring all other sensors in that node). This procedure was performed for each size of the obstacles mentioned in Table 2.

\section{Results}

The maximum detection distance for each obstacle is shown in Table 2.

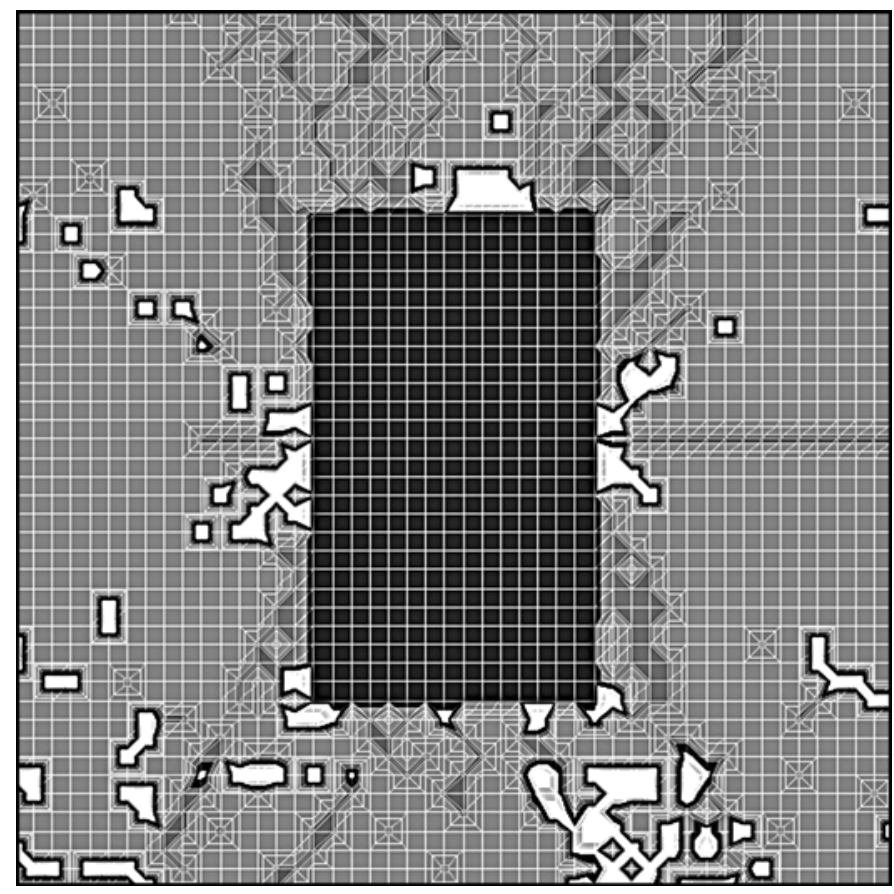

Figure 5.

Modified sensor coverage (Pride Mobility Wheelchair). Coloring is described in Figure 4.

\section{Minimum Corridor Width}

\section{Method}

The DSS was placed parallel to a wall and driven $4.6 \mathrm{~m}$ $(15 \mathrm{ft})$ while a constant distance from the wall was maintained (Figure 6). For each perpendicular distance, five trials were administered. The number of times the DSS stopped the wheelchair from moving and the time of completion were noted. If the DSS stopped the wheelchair more than five times in a single trial, that distance was considered too short and not navigable with the DSS.

\section{Results}

The mean and SD of the time to travel $4.6 \mathrm{~m}$ and the number of stops are shown in Table 3. The DSS was automatically switched to the right wall-following mode during this set of testing.

\section{Minimum Door Width}

\section{Method}

The experimental setup for minimum door-width travel is shown in Figure 7. Two $45.7 \times 10.2 \times 121.9 \mathrm{~cm}$ (18 $\times 4 \times 48$ in.) foam sheets were used to simulate doors. 
These two sheets were kept parallel in-line to each other and the distance between them was adjusted. The wheelchair began each trial $3 \mathrm{~m}$ (10 ft) away from the door opening. In forward-driving trials, the wheelchair was driven toward the door until the rear bumper passed the rear edge of the foam sheets. Five trials were administered for each

Table 2.

Maximum obstacle-detection distance.

\begin{tabular}{|c|c|c|}
\hline $\begin{array}{c}\text { Obstacle } \\
\text { Diameter }\end{array}$ & $\begin{array}{l}\text { Maximum } \\
\text { (Sonar) }\end{array}$ & $\begin{array}{l}\text { Maximum } \\
\text { (IR) }\end{array}$ \\
\hline $0.3 \mathrm{~cm}$ (1/8 in.) & $1.6 \mathrm{~m}$ (62 in.) & No detection \\
\hline $1.3 \mathrm{~cm}(1 / 2$ in.) & $2.0 \mathrm{~m}$ (81 in.) & $0.3 \mathrm{~m}$ (12 in.) \\
\hline $2.5 \mathrm{~cm}$ (1 in.) & $2.5 \mathrm{~m}$ (97 in.) & $0.9 \mathrm{~m}$ (37 in.) \\
\hline $5.1 \mathrm{~cm}$ (2 in.) & $2.7 \mathrm{~m}$ (106 in.) & $1.1 \mathrm{~m}$ (43 in.) \\
\hline $7.6 \mathrm{~cm}$ (3 in.) & $2.9 \mathrm{~m}$ (116 in.) & $1.2 \mathrm{~m}$ (49 in.) \\
\hline $15.2 \mathrm{~cm}$ (6 in.) & 3.4 m (133 in.) & $1.3 \mathrm{~m}$ (52 in.) \\
\hline $20.3 \mathrm{~cm}$ (8 in.) & $4.1 \mathrm{~m}$ (160 in.) & $1.3 \mathrm{~m}$ (52 in.) \\
\hline 30.5 cm (12 in.) & $5.4 \mathrm{~m}$ (211 in.) & $1.3 \mathrm{~m}$ (52 in.) \\
\hline $61.0 \mathrm{~cm}$ (24 in. wall) & $6.2 \mathrm{~m}$ (246 in.) & $1.5 \mathrm{~m}$ (58 in.) \\
\hline
\end{tabular}

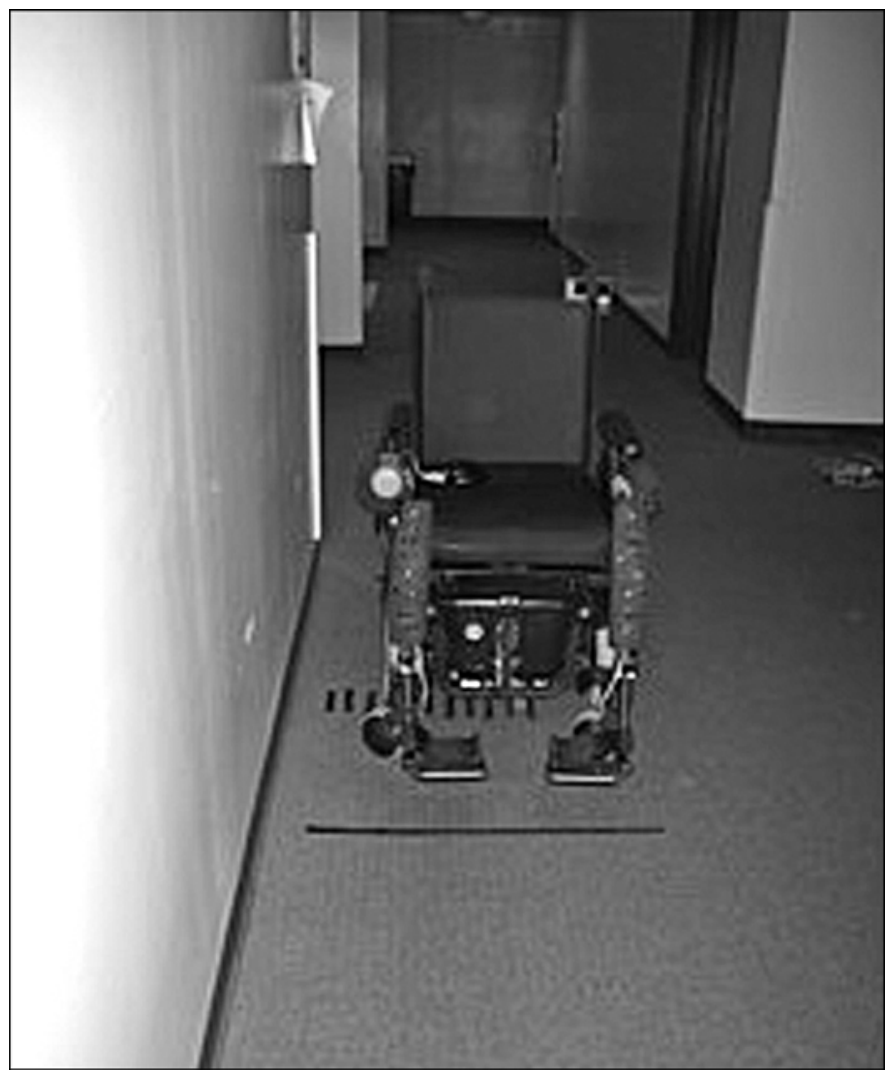

Figure 6.

Experimental setup for minimum corridor-width test. door-width setting for each direction of travel (forward and backward). The wheelchair was not allowed to go backward during forward-driving trials and vice versa. If the

\section{Table 3.}

Minimum corridor-width test results. Results are presented as mean \pm standard deviation.

\begin{tabular}{lcc}
\hline Distance from Wall & Time (s) & No. of Stops \\
\hline $10.2 \mathrm{~cm}$ (4 in.) & $52.20 \pm 4.66$ & $3.80 \pm 0.84$ \\
$15.2 \mathrm{~cm}$ (6 in.) & $12.54 \pm 1.21$ & $0.60 \pm 0.55$ \\
$20.3 \mathrm{~cm}$ (8 in.) & $8.72 \pm 0.38$ & $0 \pm 0$ \\
$25.4 \mathrm{~cm}$ (10 in.) & $8.86 \pm 0.32$ & $0 \pm 0$ \\
$30.5 \mathrm{~cm}$ (12 in.) & $6.92 \pm 1.49$ & $0 \pm 0$ \\
$35.6 \mathrm{~cm}$ (14 in.) & $7.38 \pm 0.30$ & $0 \pm 0$ \\
$40.6 \mathrm{~cm}$ (16 in.) & $7.60 \pm 0.46$ & $0 \pm 0$ \\
$45.7 \mathrm{~cm}$ (18 in.) & $7.42 \pm 0.45$ & $0 \pm 0$ \\
$50.8 \mathrm{~cm}$ (20 in.) & $7.38 \pm 0.41$ & $0 \pm 0$ \\
$55.9 \mathrm{~cm}$ (22 in.) & $7.20 \pm 0.29$ & $0 \pm 0$ \\
$61.0 \mathrm{~cm}$ (24 in.) & $7.30 \pm 0.21$ & $0 \pm 0$ \\
\hline \hline
\end{tabular}

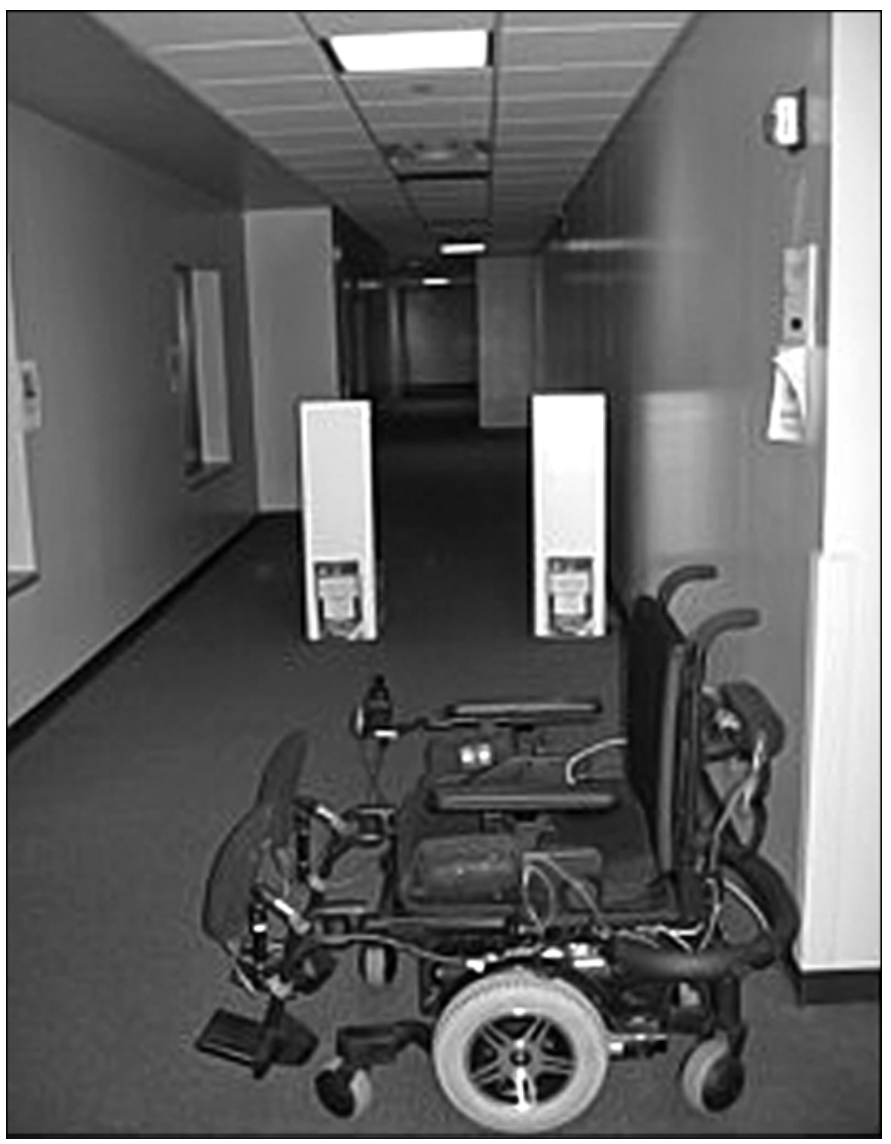

Figure 7.

Experimental setup to test minimum door width navigable by DriveSafe System. 
DSS stopped the wheelchair from passing through the doorway more than five times in a single trial, the DSS was considered to have failed to clear that doorway width.

\section{Results}

The DSS was able to detect the doorways and automatically switched to the doorway-passing mode during this testing. As shown in Table 4, the DSS was unable to pass through the doorways of width $71.1 \mathrm{~cm}$ (28 in.) or less. The DSS was able to cross doorways of width $81.3 \mathrm{~cm}$ (32 in.) or more with a 100 percent success rate driving forward. Driving backward, the wheelchair was consistently unable to cross doorways of width $91.4 \mathrm{~cm}$ (36 in.) or less. The DSS was reliably able to cross the doorways of width $111.8 \mathrm{~cm}$ (44 in.) or more with a 100 percent success rate.

\section{Maximum Safe Speed}

\section{Method}

The wheelchair was placed $3 \mathrm{~m}$ (10 ft) away from an obstacle formed by two $45.7 \times 10.2 \times 121.9 \mathrm{~cm}(18 \times 4 \times 48$ in.) foam sheets (Figure 8). The DSS was running in normal obstacle-avoidance mode during this set of testing. The wheelchair was driven at full speed toward the obstacles until the DSS stopped the wheelchair from moving forward. When the wheelchair was stopped, the minimum distance between the obstacle and the wheelchair's footrests was measured. Ten trials were administered for each speed.

\section{Results}

The mean and SD of stopping distance and the time of completion for each speed are shown in Table 5.

Table 4.

Door-crossing test results.

\begin{tabular}{lcc}
\hline \multirow{2}{*}{ Door Width } & \multicolumn{2}{c}{ Rate of Successful Attempts (\%) } \\
\cline { 2 - 3 } & Moving Forward & Moving Backward \\
\hline 71.1 cm (28 in.) & 0 & 0 \\
76.2 cm (30 in.) & 80 & 0 \\
81.3 cm (32 in.) & 100 & 0 \\
86.4 cm (34 in.) & 100 & 0 \\
91.4 cm (36 in.) & 100 & 0 \\
96.5 cm (38 in.) & 100 & 20 \\
101.6 cm (40 in.) & 100 & 40 \\
106.7 cm (42 in.) & 100 & 80 \\
111.8 cm (44 in.) & 100 & 100 \\
116.9 cm (46 in.) & 100 & 100 \\
121.9 cm (48 in.) & 100 & 100 \\
\hline \hline
\end{tabular}

\section{Bumper Sensitivity}

\section{Method}

A digital weight measuring scale was used to determine the amount of force required to activate the bumpers. The force on the bumper segments was applied to an area of $1.9 \times 3.8 \mathrm{~cm}(0.75 \times 1.50 \mathrm{in}$.). The force divided by the area was used to calculate pressure. For each bumper segment, 10 data points of activation pressure were calculated.

\section{Results}

The mean and SD for each bumper segment are shown in Figure 9. The results indicate that bumper activation pressure is not uniform across the segments. Bumper segment 5 required the lowest activation pressure (mean \pm $\mathrm{SD}=8.1 \times 10^{3} \mathrm{~Pa} \pm 206.8$ ), and bumper segment 9 required the highest activation pressure (mean $\pm \mathrm{SD}=$ $18.9 \times 10^{3} \pm 275.8$ ). The variation in activation pressure was due to variations in the tuning of a comparator circuit in the hardware. This tuning cannot be adjusted beyond a certain point because this will produce false positive activation of the bumpers.

\section{Power Consumption}

\section{Method}

The DSS architecture draws power from the underlying power wheelchair batteries. The DSS hardware can operate anywhere between 12 and $35 \mathrm{~V}$. Since the DSS hardware will always be active when a person is using his

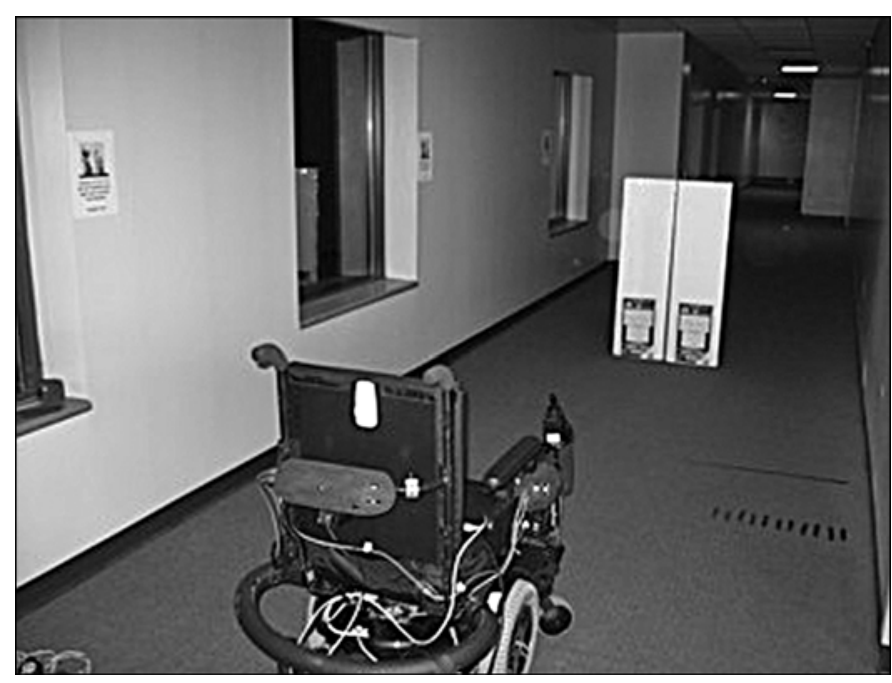

Figure 8.

Experimental setup for safe speed tests. 
Table 5.

Results from safe-speed tests. Results are presented as mean \pm standard deviation.

\begin{tabular}{crc}
\hline Speed & Time (s) & $\begin{array}{c}\text { Stopping Distance } \\
(\mathbf{c m})\end{array}$ \\
\hline $1.3 \mathrm{~km} / \mathrm{h}(0.8 \mathrm{mph})$ & $13.89 \pm 0.49$ & $42.7 \pm 1.0$ \\
$1.6 \mathrm{~km} / \mathrm{h}(1.0 \mathrm{mph})$ & $12.75 \pm 0.38$ & $41.6 \pm 1.3$ \\
$1.9 \mathrm{~km} / \mathrm{h}(1.2 \mathrm{mph})$ & $10.48 \pm 0.53$ & $40.6 \pm 2.7$ \\
$2.2 \mathrm{~km} / \mathrm{h}(1.4 \mathrm{mph})$ & $8.12 \pm 0.83$ & $40.1 \pm 2.6$ \\
$2.6 \mathrm{~km} / \mathrm{h}(1.6 \mathrm{mph})$ & $5.99 \pm 0.34$ & $38.6 \pm 2.6$ \\
$2.9 \mathrm{~km} / \mathrm{h}(1.8 \mathrm{mph})$ & $5.46 \pm 0.43$ & $38.35 \pm 2.5$ \\
$3.2 \mathrm{~km} / \mathrm{h}(2.0 \mathrm{mph})$ & $5.14 \pm 0.45$ & $31.5 \pm 2.5$ \\
$3.5 \mathrm{~km} / \mathrm{h}(2.2 \mathrm{mph})$ & $5.01 \pm 0.52$ & $26.9 \pm 2.5$ \\
$3.9 \mathrm{~km} / \mathrm{h}(2.4 \mathrm{mph})$ & $4.97 \pm 0.78$ & $21.6 \pm 4.2$ \\
$4.2 \mathrm{~km} / \mathrm{h}(2.6 \mathrm{mph})$ & $4.64 \pm 0.59$ & $10.4 \pm 1.4$ \\
$4.5 \mathrm{~km} / \mathrm{h}(2.8 \mathrm{mph})$ & $4.43 \pm 0.60$ & $3.7 \pm 3.3$ \\
\hline \hline
\end{tabular}

or her wheelchair, excessive power consumption by the DSS hardware could limit the performance and life of the underlying wheelchair. To reduce consumption of power, the DSS implements a low-power sleep state. To test power consumption, we used a benchtop power supply to supply $24 \mathrm{~V}$ to the DSS hardware and measured current using a digital multimeter.
Results

The average power consumption by the DSS hardware was $16.4 \mathrm{~W}$ when the DSS was "awake" and functioning. Maximum power consumption when all LEDs and speakers in the DSS architecture were switched on was $26.2 \mathrm{~W}$. Minimum power consumption occurred when the DSS was in sleep mode was $1.0 \mathrm{~W}$.

\section{DISCUSSION}

\section{Sensor Coverage}

Sensor coverage was achieved on all sides of the wheelchair. Most blind spots (shown in white in Figure 5) are small and isolated. In order to reach these spots, an obstacle would need to pass through areas for which sensor coverage is available, reducing the risk of a collision. Extensive blind spots exist in the rear-right corner of the wheelchair. These blind spots appear to be due to imperfections in the way the sonars are mounted in the rear sensor node and may be remedied by changing the mounting method for the rear sensor node. The blind spots appear to be due to problems in assembly or mounting rather than the design of the sensor node itself, in part because an

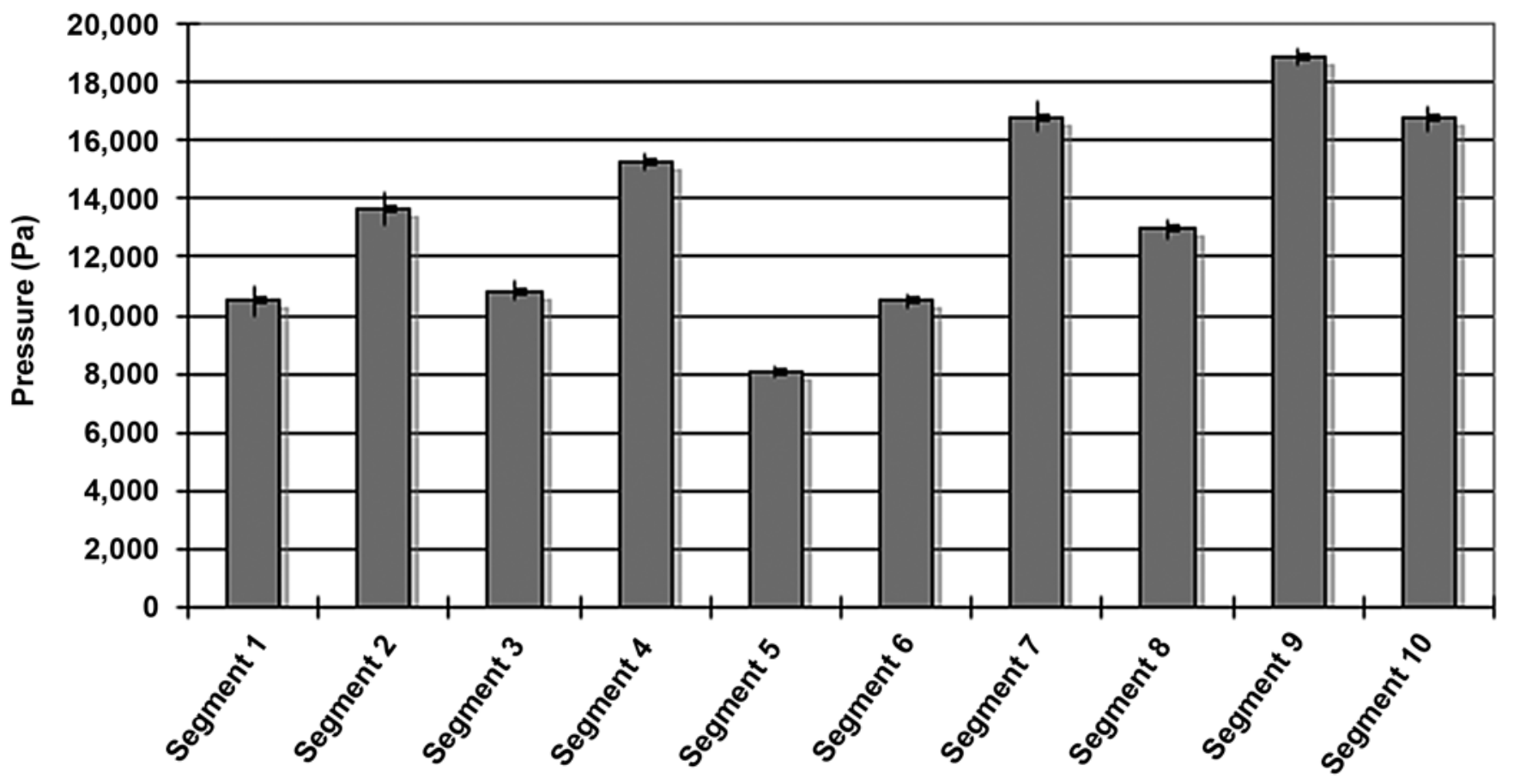

Figure 9.

Bumper activation pressure. Height of bar indicates mean pressure, vertical lines above and below line indicate one standard deviation. 
error in the node design would be expected to produce a symmetrical area of blind spots in the rear-left corner.

\section{Maximum Obstacle-Detection Distance}

The obstacle-detection ability of the DSS varies with the diameter of the obstacle. Larger obstacles can be detected further away by the sonars. Sonars could detect most obstacles at least $2 \mathrm{~m}$ away, and even the smallest obstacle was detected $1.6 \mathrm{~m}$ away, allowing time for the wheelchair to slow down and stop when an obstacle was detected.

IRs could not detect small or distant obstacles because their range is small (less than $1.5 \mathrm{~m}$ ) and their detection cone is very small compared with sonars. Not surprisingly given its resolution of $2.5 \mathrm{~cm}$, the IR sensor was unable to detect the $0.3 \mathrm{~cm}$ diameter obstacle at any distance; this obstacle was only detected by sonar. Surprisingly, the IR did detect the $1.3 \mathrm{~cm}$ diameter obstacle, although it is smaller than the nominal sensor resolution. IRs are therefore more useful for sensing the distance to nearby obstacles (especially within the minimum detection distance of the sonars), particularly if the obstacle is large (such as a doorframe or corridor wall). IRs also provide a second sensing modality for detecting moderately large surfaces that are smooth or curved and therefore might otherwise not be detected because of the characteristics of the sonar.

\section{Minimum Corridor Width}

Results from these experiments indicate that the DSS was able to follow a wall as close as $15.2 \mathrm{~cm}$ (6 in.) away without stopping. The DSS was unable to follow the wall when the distance was $10.2 \mathrm{~cm}$ (4 in.) or less. This was due to the inability of the sensors to reliably report ranges less than $15.2 \mathrm{~cm}$ (6 in.).

\section{Minimum Door Width}

The DSS was able to consistently navigate doorways of width $81.3 \mathrm{~cm}$ (32 in.) or less driving forward and $111.8 \mathrm{~cm}$ (44 in.) or less while moving backward. The DSS could not pass through narrow doorways for several reasons:

1. The sensor nodes and bumpers increased the width of the wheelchair.

2. The minimum detection distance for the sonars and IRs was too large; the smallest range value a sonar will return is $15.2 \mathrm{~cm}$ (6 in.), so it is difficult to know for certain whether an obstacle is $15.2 \mathrm{~cm}$ (6 in.) away or $2.5 \mathrm{~cm}$ (1 in.) away. Similarly, the nonlinear behavior of the IRs at short distances means they cannot determine range reliably at distances less than $20.3 \mathrm{~cm}$ (8 in.).

3 . The position of the sensors in the DSS architecture was not appropriate for detecting doors and narrow openings. The large detection cone of the sonars made finding the exact location of an opening difficult.

Moving backward, the DSS was at a greater disadvantage because the positions of the rearward-facing sonars and IRs were not appropriate to detect the doorways and trigger the door-crossing mode. Further, the large detection area of the sonars detected the door posts as obstacles, even when they were not in the direction of movement of the wheelchair.

The effect of the sensor nodes on the width of the wheelchair and the positioning of the sensor nodes (items 1 and 2 above) can be addressed through adjustments to the design of the sensor nodes. The minimum detection distance of the sensors will continue to be an issue. This issue has been addressed in the current design by triggering the door-crossing mode when the system is far enough from a doorway to reliably detect both sides and then slowing the wheelchair and relying primarily on the bumpers to allow safe passage through the doorway itself.

\section{Maximum Safe Speed}

The data in Table 5 can be compared with target desired stopping distance. For this study, a goal was set at $10.2 \mathrm{~cm}$ (4 in.) from the footrests to accommodate the front bumpers. Speeds for which the stopping distance was less than $10.2 \mathrm{~cm}$ (4 in.) were considered unsafe. The results indicate that the maximum safe speed for the current sensor sampling rate and obstacle-avoidance algorithm is $4.2 \mathrm{~km} / \mathrm{h}(2.6 \mathrm{mph})$. While this is lower than the maximum speeds available with most wheelchairs, it is 79 to 91 percent of the average comfortable walking speed depending on the population [21]. The maximum safe speed of the wheelchair can be further increased by sampling the sensors more often and further modifying the obstacle-avoidance algorithm to slow the wheelchair more rapidly in the presence of obstacles at faster wheelchair speeds.

\section{Bumper Sensitivity}

The bumpers required between $8.1 \times 10^{3} \mathrm{~Pa}$ and $18.9 \times$ $10^{3} \mathrm{~Pa}$ to register an obstacle. In our testing environment, this was adequate to trigger the bumpers when colliding with a stationary obstacle at low speeds. 


\section{Power Consumption}

The wheelchairs in this study use two gel-cell leadacid batteries, each rated for $12 \mathrm{~V}$ and $60 \mathrm{Ah}$. When drawing maximum current (26.2 W), the DSS architecture will reduce the available battery power to $58.2 \mathrm{Ah}$, a 3 percent reduction compared with the maximum rated power for these batteries. A power wheelchair travels 23.6 to $57.7 \mathrm{~km}$ on a single charge [22]. When equipped with the DSS, this wheelchair will travel $22.9 \mathrm{~km}$ to $56 \mathrm{~km}$. Use of the DSS with the wheelchair will not significantly affect the battery life or number of charges required per day.

\section{Limitations}

The sensor mounting pattern was designed to detect obstacles as small as $7.6 \mathrm{~cm}$ (3 in.) in height to overhanging obstacles at a height of $1.4 \mathrm{~m}$ (55 in.). Informal testing indicates that the DSS can detect obstacles across this range. However, this study did not formally evaluate detection of obstacles at different heights because of time constraints. More formal testing is needed to indicate sensor coverage at a variety of heights for a variety of positions surrounding the wheelchair.

The performance of IRs and sonars depends on the surface characteristics of the obstacles, ambient temperature, lighting, humidity, and air velocity [23]. The results presented here were obtained indoors at a temperature of $22^{\circ} \mathrm{C}\left(71^{\circ} \mathrm{F}\right)$ and in fluorescent lighting. Informal testing was performed with various real-world obstacles outdoors in sunlight and varying temperature and indoors in varying lighting conditions. This testing appeared to corroborate the results of this study, but more formal testing is necessary to document performance under varied obstacle properties and environmental conditions.

All tests in this study involved the wheelchair being static or moving straight forward or backward. Turning influences scan capacity as sensors sweep toward lateral blind spots. Further testing is needed with the wheelchair turning toward obstacles in various positions relative to the wheelchair.

Turning behavior of the wheelchair, and therefore performance of the collision-avoidance system, can be affected by the wheelchair drive type (rear-wheel, midwheel, or front-wheel drive). Although prior work evaluated wheelchairs in each category [20], this prototype was only evaluated on mid-wheel drive wheelchairs.

In this study, the bumpers were formally tested while the wheelchair was stationary and more informally tested with the wheelchair colliding with stationary obstacles at low speeds. Further testing will be needed in a variety of settings to ensure that the bumpers are reliably activated.

\section{Design Implications and Future Work}

The results of this study indicate some limitations of the system that can be addressed in future design iterations. For example, the system has difficulty navigating narrow doorways. It is expected that this can be mitigated somewhat in hardware (by redesigning the side and rear sensor nodes) and software (using the door-crossing mode).

The system's modular design allows it to be mounted as an aftermarket device onto a variety of wheelchair models. Therefore, the system can be integrated with the seating system, control device, and other wheelchair characteristics that are most appropriate to a particular user. Further, the system can be transferred to another wheelchair. A power wheelchair's life span varies from 3 to 5 years depending on usage and driving conditions. The DSS's add-on architecture makes it useful across multiple wheelchairs without requiring additional expenditure every time the user changes his or her wheelchair. Moreover, children with developmental disabilities can continue to use the DSS as their seating, positioning, and mobility needs change over time. For full realization of the benefits of this modular design, the system components must be sufficiently robust to survive use on multiple wheelchairs. Future testing will need to address the robustness of the modules under varied physical stresses, electromagnetic interference, and weather conditions and, thereby, to estimate an expected lifetime for the system.

The modular design of the system also allows the number of sensor nodes to vary from zero to five, depending on the sensor coverage each user requires. People with limited neck motion might only require navigation assistance while backing up, so that only the rear sensor node is needed. Individuals with hemispatial neglect may require only coverage on the side of neglect and will therefore only need either the left or right sensor node. By providing only those nodes required by the user, the system cost can be reduced. Performance testing in this study used a full complement of five sensor nodes. Further work is needed to document system performance with varying numbers and combinations of sensor nodes.

The prototype in this study acted only to slow down or stop the wheelchair. Earlier prototypes explored shared-control methods in which the wheelchair would assist with steering around obstacles [20]. For the current 
prototype, we decided to maintain the user's control of the direction of travel because of concerns that automated changes to the direction of travel could be disorienting for users with visual impairments and undesirable to some other users who may wish to maintain a higher degree of control. Future work could compare usability and user preference between the current approach and a modified system that automatically steers the wheelchair around obstacles.

Beyond performance testing of the system itself, testing is needed to evaluate the effectiveness and usability of the system with active participants. Testing is planned with blindfolded nondisabled subjects, orientation and mobility specialists, visually impaired subjects, and visually impaired wheelchair users. Some of these studies are underway [24].

\section{CONCLUSIONS}

The results of these tests indicate that a system using relatively low-cost sonar, IR, and force-sensing sensors can provide obstacle detection in the vicinity of a wheelchair, potentially enabling a collision-avoidance system for wheelchair users who have vision, upper-limb, or cognitive disabilities; simply have difficulty mastering safe wheelchair driving skills; or live or work in crowded settings. Results indicate that the DSS was able to provide a sensor coverage field around the wheelchair, gradually slow down and stop the wheelchair in the presence of an obstacle, drive reliably as close as $15.2 \mathrm{~cm}$ from an obstacle such as a corridor wall without interrupting forward movement, and not excessively drain the wheelchair batteries.

\section{ACKNOWLEDGMENTS}

\section{Author Contributions:}

Study concept and design: E. F. LoPresti, R. C. Simpson, V. Sharma. Acquisition of data: V. Sharma, L. C. Mostowy.

Analysis and interpretation of data: V. Sharma, E. F. LoPresti.

Drafting of manuscript: E. F. LoPresti, V. Sharma.

Critical revision of manuscript for important intellectual content:

R. C. Simpson.

Statistical analysis: V. Sharma.

Obtained funding: E. F. LoPresti, R. C. Simpson.

Administrative, technical, or material support: L. C. Mostowy.

Study supervision: E. F. LoPresti.
Financial Disclosures: Dr. LoPresti is the owner of AT Sciences, LLC. This study, funded by a National Institutes of Health Small Business Innovation Research grant, evaluates technology developed by AT Sciences for eventual commercialization.

Funding/Support: This material was based on work supported by a Small Business Innovation Research grant from the National Institutes of Health, National Institute of Child Health and Human Development (5R44HD040023-03).

Additional Contributions: L. Casimir Mostowy is now with Dynamics Inc, Pittsburgh, Pennsylvania.

\section{NOTES}

${ }^{\mathrm{a}}$ Quote from Simpson R, LoPresti E, Hayashi S, Nourbakhsh I, Miller D. The smart wheelchair component system. J Rehabil Res Dev. 2004;41(3B):429-42.

[PMID: 15543461]

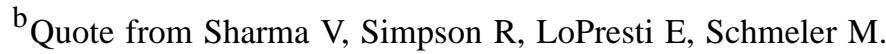
Evaluation of semiautonomous navigation assistance system for power wheelchairs with blindfolded nondisabled individuals. J Rehabil Res Dev. 2010;47(9):877-90.

[PMID: 21174252]

DOI:10.1682/JRRD.2010.02.0012

\section{REFERENCES}

1. Rosenbloom L. Consequences of impaired movement: A hypothesis and review. In: Holt KS, editor. Movement and child development. Philadelphia (PA): Lippincott; 1975.

2. Butler C. Effects of powered mobility on self-initiated behaviors of very young children with locomotor disability. Dev Med Child Neurol. 1986;28(3):325-32. [PMID: 2941328] DOI:10.1111/j.1469-8749.1986.tb03881.x

3. Brandt A, Iwarsson S, Ståhle A. Older people's use of powered wheelchairs for activity and participation. J Rehabil Med. 2004;36(2):70-77. [PMID: 15180221] DOI:10.1080/16501970310017432

4. Holt KS, editor. Movement and child development. Philadelphia (PA): Lippincott; 1975.

5. Wright BA. Physical disability, a psychosocial approach. 2nd ed. New York (NY): HarperCollins Publishers; 1983. DOI:10.1037/10589-000

6. Fehr L, Langbein WE, Skaar SB. Adequacy of power wheelchair control interfaces for persons with severe disabilities: A clinical survey. J Rehabil Res Dev. 2000;37(3):353-60. [PMID: 10917267]

7. Simpson RC, LoPresti EF, Cooper RA. How many people would benefit from a smart wheelchair? J Rehabil Res Dev. 2008;45(1):53-71. [PMID: 18566926]

DOI:10.1682/JRRD.2007.01.0015 
8. Pranghofer M. Wheels and white canes: Tips for helping blind wheelchair users. Braille Monitor. 1996;39(3).

9. Greenbaum MG, Fernandes S, Wainapel SF. Use of a motorized wheelchair in conjunction with a guide dog for the legally blind and physically disabled. Arch Phys Med Rehabil. 1998;79(2):216-17. [PMID: 9474006] DOI:10.1016/S0003-9993(98)90302-1

10. Guiding blind people who are wheelchair users. 2nd ed. London (England): Royal National Institute for the Blind; 2002.

11. Simpson RC. Smart wheelchairs: A literature review. J Rehabil Res Dev. 2005;42(4):423-36. [PMID: 16320139] DOI:10.1682/JRRD.2004.08.0101

12. Levine SP, Bell DA, Jaros LA, Simpson RC, Koren Y, Borenstein J. The NavChair Assistive Wheelchair Navigation System. IEEE Trans Rehabil Eng. 1999;7(4):443-51. [PMID: 1060932] DOI:10.1109/86.808948

13. Simpson R, LoPresti E, Hayashi S, Guo S, Ding D, Ammer W, Sharma V, Cooper R. A prototype power assist wheelchair that provides for obstacle detection and avoidance for those with visual impairments. J Neuroeng Rehabil. 2005;2:30. [PMID: 16202136] DOI:10.1186/1743-0003-2-30

14. Zeng Q, Teo CL, Rebsamen B, Burdet E. A collaborative wheelchair system. IEEE Trans Neural Syst Rehabil Eng. 2008;16(2):161-70. [PMID: 18403284]

DOI:10.1109/TNSRE.2008.917288

15. Rao RS, Conn K, Jung SH, Katupitiya J, Kientz T, Kumar V, Ostrowski J, Patel S, Taylor CJ. Human Robot Interaction: Application to Smart Wheelchairs. Proceedings of the IEEE International Conference on Robotics and Automation, 2002. Los Alamitos (CA): IEEE; 2002. p. 3583-88.

16. Farmer L. The Nurion Step Sensor: A preliminary evaluation of a wheelchair mounted system. Washington (DC): Veteran Administration Report; 1985.

17. Murarka A, Sridharan M, Kuipers B. Detecting obstacles and drop-offs using stereo and motion cues for safe local motion. Proceedings of the IEEE/RSJ International Conference on Intelligent Robots and Systems; 2008 Sep 22-26; Nice, France. Los Alamitos (CA): IEEE; 2008.

18. Nisbet P, Craig J, Odor P, Aitken S. 'Smart' wheelchairs for mobility training. Technol Disabil. 1996;5(1):49-62. DOI:10.1016/1055-4181(96)00147-1
19. Simpson RC, Poirot D, Baxter MF. The Hephaestus Smart Wheelchair system. Proceedings of the International Conference on Rehabilitation Robotics; 1999 Jul 12; Stanford, CA. Available from: http://www.rehabrobotics.org/icorr1999/ papers/.

20. Simpson R, LoPresti E, Hayashi S, Nourbakhsh I, Miller D. The smart wheelchair component system. J Rehabil Res Dev. 2004;41(3B):429-42. [PMID: 15543461] DOI:10.1682/JRRD.2003.03.0032

21. Bohannon RW. Comfortable and maximum walking speed of adults aged 20-79 years: Reference values and determinants. Age Ageing. 1997;26(1):15-19. [PMID: 9143432] DOI:10.1093/ageing/26.1.15

22. Cooper RA, Thorman T, Cooper R, Dvorznak MJ, Fitzgerald SG, Ammer W, Song-Feng G, Boninger ML. Driving characteristics of electric-powered wheelchair users: How far, fast, and often do people drive? Arch Phys Med Rehabil. 2002;83(2):250-55. [PMID: 11833031]

DOI:10.1053/apmr.2002.28020

23. LoPresti EF, Simpson RC. Miller D, Nourbakhsh Illah. Evaluation of sensors for a smart wheelchair. Proceedings of the RESNA 2002 Annual Conference; 2002 Jun; Orlando, FL.

24. Sharma V, Simpson R, LoPresti E, Schmeler M. Evaluation of semiautonomous navigation assistance system for power wheelchairs with blindfolded nondisabled individuals. J Rehabil Res Dev. 2010;47(9):877-90. [PMID: 21174252] DOI:10.1682/JRRD.2010.02.0012

Submitted for publication January 25, 2010. Accepted in revised form November 29, 2010.

This article and any supplementary material should be cited as follows:

LoPresti EF, Sharma V, Simpson RC, Mostowy LC. Performance testing of collision-avoidance system for power wheelchairs. J Rehabil Res Dev. 2011;48(5):529-44.

DOI:10.1682/JRRD.2010.01.0008

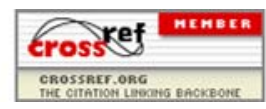


